\title{
Esophageal Neuroendocrine Tumor
}

National Cancer Institute

\section{Source}

National Cancer Institute. Esophageal Neuroendocrine Tumor. NCI Thesaurus. Code C95616.

A well differentiated, low or intermediate grade tumor with neuroendocrine differentiation that arises from the esophagus. 